\title{
Report of the Third Meeting of the Scientific Working Group on Chemotherapy of Leprosy (THELEP) of the UNDP/World Bank/WHO Special Programme for Research and Training in Tropical Diseases, Geneva, 20-22 October 1980*
}

\author{
Received for publication 21 April 1981
}

Since its first meeting in April 1977, the Scientific Working Group (SWG) on Chemotherapy of Leprosy (THELEP) of the UNDP/World Bank/WHO Special Programme for Research and Training in Tropical Diseases has promoted activities in 5 major areas: (1) field studies; (2) controlled clinical trials; (3) laboratory studies; (4) drug development studies; and (5) biological studies.

In addition, the decision was taken at the second meeting of the THELEP SWG, in March 1979, to embark on large-scale field trials of chemotherapy of lepromatous leprosy. The third meeting of the THELEYP SWG was convened, 20-22 October 1980 in Geneva, to consider progress made in these areas. In addition, the SWG reconsidered the subject of trials in non-lepromatous leprosy.

\section{Prevalence surveys of dapsone resistance}

The first cases of proven dapsone resistance, represented by relapse after many years of clinical improvement under supervised dapsone monotherapy, and despite continued therapy, were reported in 1964. Subsequently, dapsone resistance has been reported from many parts of the world. Country-wide surveys in Costa Rica and Israel reported prevalences of, respectively, $7 / 100$ and 4/100 patients at risk. More than 200 proven cases have been recognized in Malaysia. And an annual incidence of 3/100 was reported from one centre in Ethiopia. Thus, the situation as the THELEP programme was organized was one of concern. If the situation in Ethiopia were representative of large parts of the world, we faced the alarming prospect of being required to control leprosy without dapsone. If, on the other hand, the situation in Costa Rica, Israel and Malaysia (where a prevalence slightly in excess of 2/100 was calculated) were more representative, there was cause for concern but no emergency. To clarify the situation, THELEP embarked on a programme of prevalence surveys of dapsone resistance. A standard protocol for prevalence surveys of secondary (acquired) dapsone resistance was prepared, and formal surveys were initiated in Burma, two districts in South India and Upper Volta. In addition, a survey of primary resistance was initiated in the Philippines.

*In addition to this report, a further paper presented at the October 1980 meeting of the THELEP Scientific Working Group appears in this issue of Leprosy Review. It is our understanding that this report, together with other papers presented at the meeting, will appear in the December 1981 issue of International Journal of Leprosy. Editor 
The incomplete results were reported of a prevalence survey of secondary dapsone resistance in Gudiyatham Taluk, South India, where a leprosy control programme employing dapsone in full dosage and enjoying $80 \%$ attendance has been in place for 16 years. Of a total of 1,580 known patients with lepromatous leprosy, 1,431 were re-examined, of whom 46 were found to have clinical and bacteriologic evidence of relapse. Of 26 biopsied for mouse inoculation, 24 were found to harbour dapsone-resistant Mycobacterium leprae; adding 9 cases of dapsone resistance previously found in this population, the minimal estimate of prevalence is $2.3 \%$.

The results were reported of a prevalence survey of primary dapsone resistance in Cebu, Philippines, in which consecutive patients with apparently untreated, multibacillary leprosy were biopsied and mice inoculated. Of the first 50 patients, the specimens of 47 contained organisms infective for mice; the organisms were fully susceptible to dapsone in 46 of the 47 cases, low-grade resistance having been demonstrated in a single instance, yielding a prevalence of $2.1 \%$.

In discussion, it was reported that primary dapsone resistance has been recognized among patients with previously untreated multibacillary leprosy in San Francisco and Carville (USA), Burma, Malaysia, Mali and South India.

\section{Controlled clinical trials}

The results of short-term trials of a variety of single-drug regimens were reviewed. Patients' organisms were rendered non-infective for mice after treatment for 100 days with dapsone in full dosage; 150 days with clofazimine in a dosage of $200 \mathrm{mg}$ daily; about the same with clofazimine $100 \mathrm{mg} 3$ times weekly but somewhat longer with clofazimine in dosages of $300 \mathrm{mg}$ weekly, $600 \mathrm{mg}$ every 2 weeks, and $600 \mathrm{mg}$ on 2 consecutive days every 4 weeks; about 30 days on the average in 3 patients with ethionamide $250 \mathrm{mg} 3$ times daily; and 3 or 4 days with rifampicin in a variety of regimens, including $600,900,1,200$ and 1,500 $\mathrm{mg}$ in a single dose and 300 or $600 \mathrm{mg}$ daily.

Also reviewed were the results of clinical trials in which demonstration of persisting $M$. leprae by inoculation of immunosuppressed mice was attempted. In the first persister study, small numbers of viable, dapsone-susceptible $M$. leprae were detected by inoculation of normal or immunosuppressed mice in at least 1 tissue (skin, nerve, skeletal muscle or dartos) of 7 of 12 patients who had been treated with dapsone in supervised, full dosage for a minimum of 10 years. Subsequent trials revealed evidence of persisting $M$. leprae in patients treated for from 6 months to 5 years with rifampicin $600 \mathrm{mg}$ daily, clofazimine $100 \mathrm{mg}$ daily or rifampicin $600 \mathrm{mg}$ daily plus dapsone $100 \mathrm{mg}$ daily.

At the time that the THELEP programme began, it was already clear that even a single dose of rifampicin was maximally effective as measured in short-term trial with inoculation of intact mice, whereas the combination of dapsone and rifampicin, each drug administered daily in full dosage, was inadequate to eradicate the persisting $M$. leprae, detected by inoculation of immunosuppressed mice with large numbers of $M$. leprae. Recognizing the need to employ drug combinations including some dosage of rifampicin, THELEP embarked on 2 controlled clinical trials among previously untreated patients with multibacillary leprosy, in which thymectomized-irradiated mice are inoculated with as many as $10^{5}$ M. leprae per foot pad after 3, 12 and 24 months of treatment.

The regimens employed are at the Central Leprosy Teaching and Research Institute, Chingleput, South India:

$\mathrm{A}_{1}$ dapsone $100 \mathrm{mg}$ daily, rifampicin $600 \mathrm{mg}$ daily and clofazimine $100 \mathrm{mg}$ daily;

C dapsone $100 \mathrm{mg}$ daily plus rifampicin in a single initial dose of $1,500 \mathrm{mg}$;

$\mathrm{D}_{1}$ dapsone $100 \mathrm{mg}$ daily plus rifampicin in a single initial dose of $1,500 \mathrm{mg}$ plus 
clofazimine in an initial 3-month course of $100 \mathrm{mg}$ daily;

and at the Institut Marchoux, Bamako, Mali:

$\mathrm{A}_{2}$ dapsone $100 \mathrm{mg}$ daily, rifampicin $600 \mathrm{mg}$ daily and prothionamide $500 \mathrm{mg}$ daily;

$\mathrm{C}$ as for Chingleput;

$\mathrm{E}_{2}$ dapsone $100 \mathrm{mg}$ daily plus initial 3-month courses of rifampicin $900 \mathrm{mg}$ weekly and prothionamide $500 \mathrm{mg}$ daily.

About 48 patients have been recruited into the trial at Chingleput, which began recruiting patients in August 1978.

One patient was found to have low-grade dapsone resistance in the pretreatment biopsy specimen, and another patient's organisms failed to infect mice pretreatment. The treatment regimens have been well-tolerated; clinical and bacteriological improvement has been gratifying; the results of inoculation of immunosuppressed mice are as yet too fragmentary to permit analysis.

Thirty-one patients have been admitted into the trial at Bamako since November 1978. Organisms from the pretreatment specimen of 1 patient did not infect mice, and those from the pretreatment specimens of 5 patients exhibited low-grade resistance to dapsone. The results of inoculation of immunosuppressed mice were too fragmentary to permit analysis.

\section{Work with immunosuppressed rodents}

The papers in this segment of the meeting were devoted to a review of work in progress on immunosuppressed rodents, with particular reference to the detection of persisting M. leprae. Originally, thymectomy of mice was performed at a few weeks of age; a few weeks later, the animals were subjected to whole-body irradiation with $900 \mathrm{R}$, followed immediately by bone marrow replacement. This animal permitted multiplication of $M$. leprae well above the ceiling in normal mice of about $10^{6}$ organisms per foot pad; in addition, systemic spread of the infection and grossly-evident lesions were common. More recently, decreased survival of these 'T900R' mice has required a change of procedure; mice are now thymectomized as before, and subsequently subjected to $200 \mathrm{R} 5$ times at intervals of 2 weeks ('T $200 \times 5 \mathrm{R}$ '). This animal does not require bone marrow replacement, but its immunity is not suppressed to the same degree as is that of the T900R mouse. This animal has been useful in attempts to detect persisting $M$. leprae in biopsy specimens obtained from patients under treatment, and is currently being applied to the ongoing THELEP controlled clinical trials in Bamako and Chingleput.

The results were received of work with neonatally-thymectomized rats (NTR), which are thymectomized within 16 hours of birth and either inoculated intravenously with large numbers of $M$. leprae, to be used subsequently in chemotherapeutic experiments, or for the detection of persisters, either in patients or in $M$. leprae-infected NTR undergoing chemotherapy.

The objective of the chemotherapeutic experiments has been to reproduce in NTR the state of microbial persistence encountered in patients with lepromatous leprosy, and to devise drug regimens that are active against these persisting $M$. leprae. It has been found that 10 daily doses of rifampicin, each $10 \mathrm{mg} / \mathrm{kg}$ body weight, administered on a background of dapsone administered continuously in a concentration in the diet of $10^{-5} \mathrm{~g} \%$, the minimal effective dose, produces a situation comparable to that seen in patients: subinoculation of small numbers of $M$. leprae to normal mice fails to result in multiplication of the organisms, whereas subinoculation of larger numbers to NTR results in multiplication.

The incomplete results were presented of a clinical trial in which 2 regimens are being compared: daily dapsone plus rifampicin either in a single initial 1,500 $\mathrm{mg}$ dose or in a 
once-weekly $900 \mathrm{mg}$ dose. Skin biopsy specimens are obtained 3 or 4 days, and 1, 2 and 4 weeks after the start of treatment. Normal mice are inoculated with 5,000 AFB per foot pad, and NTR are inoculated with $10^{5}-10^{7}$ AFB per foot pad. The results appear to confirm the usefulness of the NTR in detecting the presence of persisting $M$. leprae.

Early work was described with the congenitally athymic rat, which is more effectively and more uniformly immunosuppressed than is the NTR. Although the athymic rat appears to possess active non-T-cell mediated mechanisms that account for its ability to survive under conventional conditions of husbandary, it nevertheless appears to permit multiplication and dissemination of $M$. leprae from large inocula.

The results were reviewed of work in which gnotobiotic nude (hairless, congenitallyathymic) mice are infected with $M$. leprae. Intravenous, intraperitoneal and subcutaneous (in the foot pad) inoculation of the organisms appears to result in disseminated infection, and animals inoculated in the foot pad exhibit some swelling of the feet some months later. A comparison of nude, T200 $\times 5 \mathrm{R}$, and normal mice inoculated with $10^{2}$ fresh $M$. leprae diluted by large numbers of irradiated organisms established the superiority of the nude mouse over both of the others, in terms of permitting multiplication of the viable organisms.

\section{Studies of patient compliance}

The background was reviewed of our concern with the compliance of patients with chemotherapy-particularly chemotherapy with dapsone, which is traditionally self-administered. Studies carried out in several parts of the world have demonstrated that compliance of leprosy patients with self-administered dapsone as monotherapy ranged from very poor to poor. The focus of THELEP has been the development of simpler, more specific means of monitoring compliance under field conditions.

The Bratton-Marshall colorimetric method applied to urine has been most widely used. Simultaneous assay of urinary creatinine makes the test more precise, but there remains an important element of lack of specificity for dapsone. An alternative is the addition to dapsone tablets of a readily-detected marker. Such a marker is a small quantity $(6 \mathrm{mg})$ of isoniazid, which can be detected by a simple and specific test. Use of an isoniazid marker would permit recognition of dapsone ingested within the 16 hours preceding the urine collection. Most desired, however, is a specific test that will detect dapsone ingestion during the preceding 5-10 days. After 5 consecutive missed daily $100 \mathrm{mg}$ doses of dapsone, the plasma dapsone concentration has fallen below that required to inhibit multiplication of low-resistant $M$. leprae; after 10 consecutive missed daily doses, the plasma dapsone concentration is insufficient to inhibit multiplication of $M$. leprae fully susceptible to dapsone.

The results of work based on two 'ELISA' techniques were described. Both techniques are capable of detecting dapsone in $\mathrm{ng} / \mathrm{ml}$ concentrations. The less sensitive technique is capable of recognizing dapsone in the urine as late as 8 days after a single $100 \mathrm{mg}$ dose; the more sensitive technique can detect dapsone in a finger-prick blood specimen as late as 5 days after a dose. These techniques have been made available as 'kits', and have been employed by leprosy workers in endemic areas.

\section{Drug development}

Work in progress on clofazimine analogues in 3 laboratories was reviewed. A mass of data has been generated on structural requirements for activity against Mycobacterium sp. 607, acute toxicity in the mouse, and mutagenesis; these have been submitted for analysis of the 
quantitative structure-activity relationships (QSAR). The 3 major pigmented metabolites in human urine have been separated and identified; these were found not to be mutagenic.

The results of work with cell-free preparations of folate-synthesizing enzymes of $\mathrm{Myco}$ bacterium lufu and $M$. leprae were reviewed. The action of dapsone in these systems appears to be qualitatively as well as quantitatively different from that in the comparable Escherichia coli system. Dapsone is much more potent in the 2 mycobacterial systems. Also, dapsone is incorporated in the $E$. coli system into an analogue of the product of the reaction between dihydropteridine alcohol and $p$-aminobenzoic acid, whereas no analogue is formed in either mycobacterial system.

Work was described on 2 systems that involve the uptake by $M$. leprae in short-term culture (in macrophages or in vitro) of various radioisotope-labelled substrates. These systems show promise of usefulness in screening drugs for activity against $M$. leprae, and in testing the drug-susceptibility of $M$. leprae.

\section{Trials of chemotherapy of non-lepromatous leprosy}

Trials of chemotherapy of non-lepromatous leprosy have been an objective of high priority since the THELEP Programme was first established. Yet, no such trials have been undertaken, because of the difficulties inherent in measuring the response to therapy of nonlepromatous leprosy, in which $M$. leprae are too few to permit animal inoculation, and also because it appeared more urgent to develop better methods for the control of lepromatous leprosy, the more infectious form of the disease. Because it appeared important to reconsider the priority of these trials within the framework of THELEP activities, a segment of the meeting was devoted to reviews of the importance of non-lepromatous leprosy in leprosy control programmes, and of the methods that have been employed in several trials in the past.

The deformities that characterize non-lepromatous leprosy, which accounts for twothirds of the world's leprosy patients, have an enormous economic impact. In addition, it cannot be stated that these patients are non-infectious; the attack rate among household contacts to non-lepromatous patients was 3.7 times that among non-contacts in South India, and 1.6 times that among non-contacts in Burma. Finally, a study in Burma revealed that nearly half of the lepromatous borderline cases had evolved from 'paucibacillary' forms of the disease. On the basis of this evidence, control of non-lepromatous leprosy with more effective, short-course regimens appeared to make sense in the framework of leprosy control programmes. Merely preventing deformity and permitting much earlier 'release from control' would free enormous resources that could then be applied to the detection and treatment of patients with lepromatous leprosy.

The results of past trials were then reviewed. The most useful measurement appeared to be that of relapse after initial response and subsequent cessation of therapy. Several short-course regimens of dapsone plus rifampicin appeared undoubtedly to have been effective. And it was noted with considerable interest that, in at least 1 trial of a shortcourse regimen, clinical improvement continued even after cessation of treatment.

\section{Recommendations and conclusions}

The SWG felt that it was important to prevent the emergence of strains of $M$. leprae resistant to rifampicin and the thioamides as well as to dapsone. For this reason, every newly-discovered patient with multibacillary leprosy should be treated with combined chemotherapy, and additional drug(s) should be employed in the case of such patients already receiving dapsone as monotherapy. 


\section{THELEP Report}

It was recommended that THELEP conduct field trials of combined drug regimens designed to prevent drug resistance. In addition, THELEP should abandon its programme of formal surveys of the prevalence of secondary dapsone resistance, because those in progress appear already to have accomplished THELEP's purpose-namely, to demonstrate the widespread nature of this problem. In place of the programme of formal surveys, THELEP should promote the establishment of mouse-foot-pad laboratories around the world, and encourage them to monitor primary resistance within leprosy control programmes.

\section{Work in immunosuppressed rodents}

The SWG was of the opinion that the nude mouse and the immunosuppressed rat are unlikely to replace the thymectomized-irradiated mouse as a means of detecting $M$. leprae. On the other hand, these other immunosuppressed animals should prove extremely valuable as models of the lepromatous patient for chemotherapeutic studies.

\section{Drug development}

The SWG recommended that efforts be continued to improve the existing drugs, in terms of potency, selectivity, convenience (supervisability) and cost. In addition, THELEP should press forward in its attempts to develop screening systems other than that of the mouse foot pad. $M$. lufu has already proved so useful as a model of $M$. leprae for studies of drugs acting on folate-synthesizing enzymes as to suggest the usefulness of other model organisms that might be employed in studies of other drug classes.

\section{Chemotherapy of non-lepromatous leprosy}

The SWG felt the issue to be of sufficient urgency to require that the next step be takennamely, development of a protocol for chemotherapy trials in non-lepromatous leprosy. 